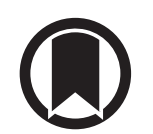

CrossMark

\title{
New understanding in the treatment of cough (NEUROCOUGH) ERS Clinical Research Collaboration: improving care and treatment for patients with cough
}

\author{
Lorcan McGarvey ${ }^{1}$, Lieven Dupont ${ }^{2}$, Surinder S. Birring ${ }^{3}$, Jeanette Boyd ${ }^{4}$, \\ Kian Fan Chung ${ }^{5}$, Marta Dabrowska ${ }^{6}$, Christian Domingo ${ }^{7}$, Giovanni Fontana ${ }^{8}$, \\ Laurent Guilleminault ${ }^{9}$, Peter Kardos (10) ${ }^{10}$, Eva Millqvist ${ }^{11}$, Alyn H. Morice ${ }^{12}$, \\ Jaclyn A. Smith ${ }^{13}$, Jan Willem van den Berg ${ }^{14}$ and Charlotte Van de Kerkhove ${ }^{15}$ \\ on behalf of the NEUROCOUGH Clinical Research Collaboration ${ }^{16}$
}

\begin{abstract}
Affiliations: ${ }^{1}$ Centre for Experimental Medicine, School of Medicine, Dentistry and Biomedical Sciences, Queen's University Belfast, Belfast, UK. ${ }^{2}$ Dept of Respiratory Diseases, University Hospital Leuven, Katholieke Universiteit Leuven, Leuven, Belgium. ${ }^{3}$ Centre for Human and Applied Physiological Sciences, School of Basic and Medical Biosciences, King's College London, London, UK. 'European Lung Foundation, Sheffield, UK. ${ }^{5}$ National Heart and Lung Institute, Imperial College London, London, UK. ${ }^{6}$ Medical University of Warsaw, Warsaw, Poland. ${ }^{7}$ Servicio de Neumología, Hospital Parc Taulí, Sabadell, Autonomous University of Barcelona (UAB), Barcelona, Spain. ${ }^{8}$ Dept of Experimental and Clinical Medicine, University of Florence, Florence, Italy. 'Service de pneumologie-allergologie, pôle des voies respiratoires, hôpital Larrey, and Center for Pathophysiology Toulouse Purpan, INSERM U1043, CNRS UMR 5282, Toulouse III University, Toulouse, France. ${ }^{10}$ Centre for Allergy, Respiratory and Sleep Medicine at Red Cross Maingau Hospital, Frankfurt, Germany. ${ }^{11}$ Dept of Allergology, Institution of Internal Medicine, The Sahlgrenska Academy at University of Gothenburg, Gothenburg, Sweden. ${ }^{12}$ Hull York Medical School, Castle Hill Hospital, Hull, UK. ${ }^{13}$ Division of Infection, Immunity and Respiratory Medicine, University of Manchester, Manchester, UK. ${ }^{14} \mathrm{Dept}$ of Pulmonology, Isala Hospital, Zwolle, The Netherlands. ${ }^{15}$ Universitair Ziekenhuis Leuven, Leuven, Belgium. ${ }^{16} \mathrm{~A}$ full list of the members of the NEUROCOUGH Clinical Research Collaboration can be found at the end of this article.
\end{abstract}

Correspondence: Lorcan McGarvey, Centre for Experimental Medicine, School of Medicine, Dentistry and Biomedical Sciences, Queen's University Belfast, Belfast, BT9 7BL, UK. E-mail: l.mcgarvey@quub.ac.uk

@ERSpublications

The NEUROCOUGH ERS Clinical Research Collaboration brings together clinicians, scientists, patients and industry from Europe and beyond, with a focus on improving care and treatment for patients with chronic cough http://bit.ly/2Q610ER

Cite this article as: McGarvey L, Dupont L, Birring SS, et al. New understanding in the treatment of cough (NEUROCOUGH) ERS Clinical Research Collaboration: improving care and treatment for patients with cough. Eur Respir J 2019; 53: 1900787 [https://doi.org/10.1183/13993003.00787-2019].

\section{Introduction}

Chronic cough is a common and troublesome clinical problem and currently there are no effective treatments [1]. While individual specialist cough clinics have been set up in some European countries, there is no formal mechanism to develop common management approaches. Furthermore, the vast majority of clinical trials of novel anti-tussive treatment have been conducted in a limited number of sites in the UK and USA, with little in the way of cough clinical trial infrastructure across Europe [2-7]. The NEw Understanding in the tReatment Of COUGH (NEUROCOUGH) Clinical Research Collaboration (CRC) 
seeks to address this through creating a platform allowing clinicians, together with researchers in academia and industrial partners across Europe and beyond, to exchange ideas and facilitate collaborations geared towards improved care and treatment for patients with cough. The core aims of NEUROCOUGH are to: 1) create a registry of Europe-wide specialist cough clinics operating according to agreed and standardised protocols; 2) establish a Europe-wide registry of "clinical trial ready" chronic cough patients suitable for multicentre experimental medicine studies and later phase precision medicine clinical trials; 3) seek public engagement to provide input into NEUROCOUGH based on the priorities and unmet needs of patients; and 4) encourage early career researchers and clinicians into the field of cough.

In time, we envisage that NEUROCOUGH will bring clinicians, scientists, patients and industry together for larger-scale cough projects in a way that to date has not been possible. NEUROCOUGH will place Europe at the forefront of clinical improvements in chronic cough and provide a strong platform for attracting major clinical trials of anti-tussives, thus speeding up drug discovery with the ultimate aim of providing better treatments for patients with chronic cough.

\section{What is the clinical rationale for NEUROCOUGH?}

Chronic cough, defined as one persisting for more than 8 weeks, is among the most common clinical problems encountered by doctors in both general and hospital practice [1]. Chronic cough is recognised globally as an important clinical problem and to address the extent of this, a number of the NEUROCOUGH researchers undertook a review of over 10000 patients to determine the demographic profile attending specialist cough clinics worldwide [8]. Particularly evident from this study were the characteristics of patients seeking specialist help for cough. In many cases the cough had persisted for years and with no effective treatments available, many such patients are left frustrated, with their clinicians unsure how best to manage this condition. To understand the scope of the problem more completely, the NEUROCOUGH researchers also undertook a survey in collaboration with the European Lung Foundation (ELF) involving almost 2000 people throughout Europe living with chronic cough. Key findings were that the cough impacted considerably on patients' daily-life activities, with many reporting deterioration in health-related quality of life and feeling fed-up and depressed [9]. Many of those surveyed reported a complete lack of awareness among their primary care physicians of cough as a distinct clinical problem and reported seeing numerous clinicians and undergoing many failed trials of therapy. While there is some consensus on the use of systematic protocols for the diagnosis and treatment of chronic cough, standardisation across centres is variable [10]. Furthermore, chronic cough can exist as a distinct clinical entity termed "refractory", "unexplained" or "idiopathic" chronic cough, when no associated cause is apparent, or treatment of any associated causes is ineffective.

Cough can also be a prominent and troublesome symptom for patients with common lung conditions including asthma, chronic obstructive pulmonary disease (COPD), idiopathic pulmonary fibrosis (IPF) and bronchiectasis. Cough is also considered as one of the potential treatable traits of airway disease and acknowledging this more widely may help clinicians adopt a more individualised approach to treating their patients [11]. For example, in asthma, patients with cough are often given oral corticosteroids; however, this may not deliver adequate control and exposes patients to the unacceptable side-effects of high-dose steroids. In COPD, cough is reported in $70 \%$ of patients [12], and many consider it to be extremely severe [13], contributing to impaired quality of life [14]. Cough is also a central clinical feature of bronchiectasis, and during an exacerbation the cough can become much worse and contribute to impaired health status [15]. In sarcoidosis, chronic cough is a common symptom and is associated with a heightened cough reflex [16]. In IPF, a chronic dry cough is as troublesome as breathlessness and remains difficult to treat [17]. Chronic cough remains difficult to manage and many patients self-medicate with "over the counter" (OTC) cough therapies, despite a lack of evidence supporting their efficacy [18, 19]. Current estimates suggest that almost 3 billion Euros are spent annually throughout Europe on OTC therapies that have little anti-tussive effect [20].

\section{How can NEUROCOUGH improve mechanistic understanding of cough?}

There is a growing recognition that chronic cough is a heterogeneous condition in terms of its presentation and causes and the underlying mechanisms are poorly understood, representing a significant knowledge gap [21]. A number of the NEUROCOUGH researchers have been instrumental in developing the concept that the common pathophysiological mechanism of cough, regardless of the aetiology, is an inflammation-induced injurious effect of the nervous system (neuro-inflammation). This leads to a cough hypersensitivity syndrome whereby neural pathways (in the airway and the brain) become damaged by factors including viral infection, and physical and chemical irritants [22, 23]. This neural damage (neuropathy), analogous to that driving some forms of chronic pain, is central to the clinical problem of cough. The challenge is in defining the complex and heterogeneous neuronal mechanisms responsible, to help identify targets for suppressing problematic cough whilst maintaining the protective cough that is 
essential for normal lung health. Recent work has led to greater understanding of the fundamental mechanisms of cough pathophysiology [24-28] and recent international collaboration through a European Respiratory Society (ERS) initiative has led to the innovative concept of cough hypersensitivity syndrome [23]. While evidence for this concept needs to be strengthened, the emerging potential in this field is underpinned by recent and remarkable progress in novel target discovery in neurobiology [2], highlighting the timely nature of the NEUROCOUGH initiative.

\section{How can NEUROCOUGH help to deliver better treatments for cough?}

A key unmet need is the lack of effective anti-tussives and, to date, assessment of novel targets in early "proof of concept" clinical studies has come with mixed success. Although efficacy has been observed with gabapentin [29] and the blocker of the ATP receptor, P2X3 [2, 7], in subsets of cough patients, compounds directed at a range of potentially promising targets including TRPV1 [3], TRPA1 [4] and voltage-gated sodium channels [6] have failed. This has raised two important issues. First, can we improve the predictive value of existing pre-clinical cough model systems? In this regard, it is our ambition that partnership activities initiated through NEUROCOUGH will pave a way forward to enhance translation of basic discoveries into clinical success. Secondly, how can we identify the genetic and biological characteristics that define the subgroups of "responsive" cough patients to novel anti-tussive therapies? Establishing a pan-European registry of well-phenotyped patients as detailed in our second core objective (see later section) will provide future capacity for multicentre studies to determine pathophysiological mechanisms and enable translation into later phase precision medicine clinical trials.

\section{Why do we need NEUROCOUGH now?}

Europe's cough researchers and clinicians have no formal mechanism to develop common investigative and management approaches or to facilitate collaboration either with each other or with partners from industry. Individual centres of cough expertise have been set up in many countries across Europe but, to date, there has been no means to standardise and harmonise the activity in these specialist clinics. In simple terms, we have no idea currently how patients with chronic cough are evaluated and managed between countries within Europe. In addition, while there have been efforts to provide consensus on the optimal management strategies, this has been largely at an individual national level, e.g. in Germany, Spain, the UK and France [30-33]. Recently an ERS Task Force on Cough has been established, with the first meeting of members held at the 2017 ERS Scientific Assembly in Milan, Italy, and the resulting publication soon to be published. A number of Task Force members are National Leads for NEUROCOUGH and will champion the Task Force recommendations within their respective countries. Finally, and perhaps most importantly, the vast majority of single- and multicentre clinical trials of novel anti-tussives have, to date, been conducted in the UK and USA [2-7]. As the number of potential novel compounds for cough is growing and the need to conduct large phase III studies of the most promising compounds is pressing, there is an opportunity for Europe to play a leading role as the ideal environment for such studies by creating a unified approach to patient management and phenotyping. To deliver on this objective, the NEUROCOUGH CRC will support the establishment of new specialist cough clinics, creating much-needed clinical trial infrastructure throughout Europe.

In summary, recent advances in mechanistic understanding of cough, coupled with interest in its neurobiology and the development of novel therapeutic options, have provided the perfect environment in which to establish a CRC for cough. We strongly believe the NEUROCOUGH network will advance cough research and clinical management throughout Europe and generate a framework for future clinical trials.

\section{NEUROCOUGH objectives}

NEUROCOUGH seeks to achieve the following four core objectives.

\section{Objective 1: Quality improvement in clinical care}

To address the under-provision of clinical expertise in cough so evident in our patient survey, we will undertake a real-world questionnaire-based survey to accurately record how clinicians across the first wave of NEUROCOUGH partner sites manage patients with chronic cough. The nature and extent of discordance between sites will be reviewed, and consensus on core assessment protocols will be reported and serve as a template for those seeking to set up new specialist clinics in the future.

\section{Anticipated output}

The creation of a registry of Europe-wide specialist cough clinics, operating according to agreed and standardised protocols, which will be incorporated into future guidelines. 
Objective 2: Establish a chronic cough patient registry

We aim to establish the first Europe-wide chronic cough patient registry comprising pre-specified clinical, physiological and biological data. An electronic data collection form for all these variables is being developed in conjunction with the Health Informatics Centre based at the University of Dundee (UK). NEUROCOUGH will generate a registry of carefully characterised chronic cough patients (including those with refractory and unexplained cough) at existing first-wave sites (figure 1) and then, through an "open-door" policy, encourage second-wave cough centres across Europe to join NEUROCOUGH and enter data.

\section{Anticipated output}

This resource will provide, for the first time, a Europe-wide registry of "clinical trial ready" chronic cough patients suitable for multicentre experimental medicine studies and later phase precision medicine clinical trials.

\section{Objective 3: Public engagement}

With support from ELF, a cough Patient Advisory Group (PAG) is currently being established to provide input into NEUROCOUGH's design and implementation, based on the priorities and unmet needs of patients. The PAG will support the dissemination of the NEUROCOUGH outputs by providing resources for patients about diagnosis and treatment options, and through public awareness activities. Specific attention will be given to improving awareness of cough amongst primary care clinicians through links with ERS Assembly 1. The PAG will provide perspective on study design and recruitment plans, assist with developing patient information sheets and consent forms, and improve ways to recruit participants to clinical trials.

\section{Anticipated output}

Dissemination and communication of the importance of diagnosing and treating chronic cough.

\section{Objective 4: Enhance training and research capacity}

NEUROCOUGH will provide the ideal platform, in collaboration with the ERS Research Agency and Assembly 5, to run themed postgraduate workshops led by expert clinical and scientific partners to

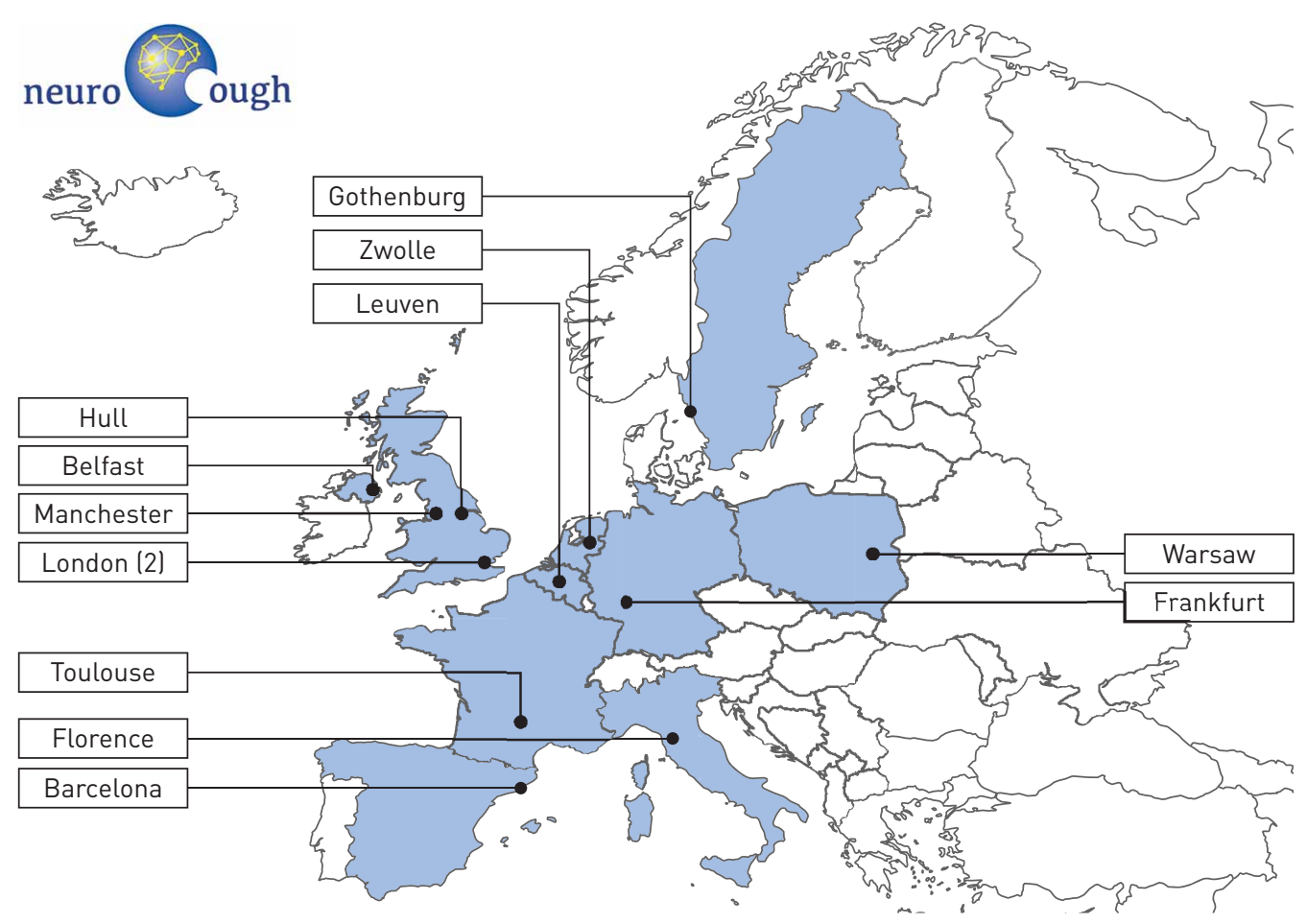

FIGURE 1 New understanding in the treatment of cough (NEUROCOUGH): first-wave specialist cough centres participating in the Europe-wide registry of chronic cough patients. 
encourage clinicians and researchers in the early part of their career to form a specialist interest in cough. We anticipate that, in time, NEUROCOUGH would seek support for research and training fellowships to undertake innovative projects based on access to the NEUROCOUGH clinical registry and any future associated biobanks. The output from the planned activities will be available to all through a comprehensive communication strategy.

Anticipated output

Encouragement of early career researchers and clinicians into the field of cough.

\section{How will NEUROCOUGH achieve its objectives?}

NEUROCOUGH has assembled a multidisciplinary team of leading cough specialist clinicians, together with clinical and basic science researchers from both academia and industry, to work with patients and the public in an integrated fashion.

The NEUROCOUGH CRC is composed of a Steering Committee comprising the co-chairs, three members of the Committee of National Leads (rotating on an annual basis), a member of the PAG and the Early Career Member responsible for the oversight of the CRC as well as for reporting to ERS. The Steering Committee will work closely with the appointed representatives from each of the industry partners. A NEUROCOUGH Registry Scientific Committee responsible for the running of the registry will be developed. The Registry Scientific Committee will have direct responsibility for the conduct of the registry, including ensuring compliance with the protocol. The Registry Scientific Committee will work with the Steering Committee to provide direction on the strategic development of the registry and the monitoring of core activities (figure 2).

\section{Looking forwards with NEUROCOUGH}

It is anticipated that NEUROCOUGH, in collaboration with the ERS Research Agency, will seek external funding support to develop a programme of work designed to address the research priorities in cough and the unmet needs in the development of novel anti-tussives. A number of these are listed in table 1. Furthermore, there is a need to raise awareness of cough as an important and difficult-to-manage clinical problem in lung diseases including COPD, asthma, IPF and bronchiectasis. The NEUROCOUGH CRC envisages fruitful collaborations with others within the ERS CRC family, including but not limited to EMBARC [34], SHARP [35], CADSET [36] and Ariane-IPF [37].

\section{Conclusion}

The NEUROCOUGH CRC brings together, for the first time, clinicians, scientists, patients and industry, to work in partnership with a vision to advance clinical management of chronic cough throughout Europe, enhance research capability and generate a framework for clinical trials. The purpose of this editorial is to inform and invite all who are interested in the field of cough to join us in achieving these goals.

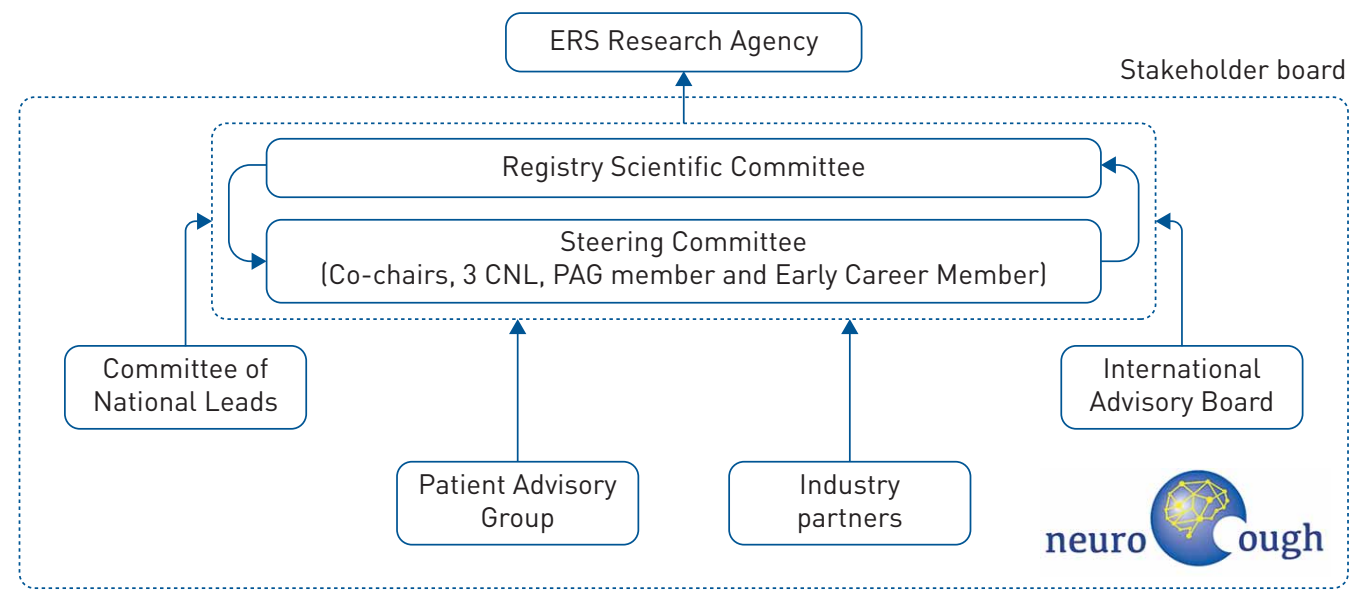

FIGURE 2 New understanding in the treatment of cough (NEUROCOUGH) structure: an operational model for the Clinical Research Collaboration. ERS: European Respiratory Society; CNL: members of the Committee of National Leads; PAG: Patient Advisory Group. 
TABLE 1 New understanding in the treatment of cough (NEUROCOUGH): future objectives

Future objectives for NEUROCOUGH
Improve understanding of the nature,
characteristics and burden of cough as a
clinical problem in primary care
Highlight the importance of cough with
clinicians working in other respiratory
disease areas (e.g. COPD, IPF, non-CF
bronchiectasis) as well as with specialists
from other clinical fields (gastroenterology,
ENT, primary care, speech and language)

\section{Address the unmet needs in development of new drugs for cough}

How will NEUROCOUGH achieve this?

Undertake a retrospective, observational analysis of electronic medical records from a large, longitudinal, anonymised primary care database with support from the Respiratory Effectiveness Group

Explore opportunities to undertake cough-focused analysis of multilevel data obtained by other members of the ERS CRC family le.g. EMBARC, SHARP, CADSET, Ariane-IPF)

Undertake a survey of how cough presents and is managed by specialists from other clinical fields (gastroenterology, ENT, primary care, speech and language)

Themed multidisciplinary workshops to address areas of unmet need: 1) refine existing and develop new methods to measure cough and cough hypersensitivity; 2) improve the predictability of human and animal models of cough for target discovery; 3) optimise design of clinical trials of novel anti-tussives

\section{Anticipated output for NEUROCOUGH}

The first comprehensive analysis of the demographic and epidemiological pattern of cough, to measure the healthcare burden in a "real-life" patient setting

A more complete understanding of the clinical impact of cough across respiratory and non-respiratory disease areas, to improve the quality of care for patients

Improved translation of basic science discoveries into clinical success

COPD: chronic obstructive pulmonary disease; IPF: idiopathic pulmonary fibrosis; CF: cystic fibrosis; ENT: ear, nose and throat; ERS: European Respiratory Society; CRC: Clinical Research Collaboration; EMBARC: European Multicentre Bronchiectasis Audit and Research Collaboration; SHARP: Severe Heterogeneous Asthma Research collaboration, Patient-centred; CADSET: Chronic Airway Diseases Early Stratification.

Acknowledgements: We are grateful to Elise Heuvelin, Celine Genton and Steve Sealy from the European Respiratory Society executive office (Lausanne, Switzerland) for their logistical support for NEUROCOUGH. We thank Tracie Andreasson (Queen's University Belfast, Belfast, UK) for clerical support.

Members of the NEUROCOUGH Clinical Research Collaboration are as follows. Chair: Lorcan McGarvey; co-chair: Lieven Dupont. National Leads: Surinder S. Birring (UK), Kian Fan Chung (UK), Marta Dabrowska (Poland), Christian Domingo (Spain), Giovanni Fontana (Italy), Laurent Guilleminault (France), Peter Kardos (Germany), Eva Millqvist (Sweden), Alyn H. Morice (UK), Jaclyn A. Smith (UK) and Jan Willem van den Berg (the Netherlands). Early Career Member: Charlotte Van de Kerkhove (Belgium). European Lung Foundation: Courtney Coleman and Jeanette Boyd. International Advisory Board: Ian Adcock (UK), Peter Dicpinigaitis (USA), Piero Geppetti (Italy), Peter Gibson (Australia), Kefang Lai (China), Stuart Mazzone (Australia), Clive Page (UK), Ian Pavord (UK) and Woo-Jung Song (South Korea).

Conflict of interest: L. McGarvey reports grants from European Union Interreg VA Health and Life Science Programme, NC3R, Chiesi, Boehringer Ingelheim and GlaxoSmithKline; personal fees (including for advisory boards) from Merck and Co., Inc., Sanofi and Bellus Health; and non-financial support from Chiesi, Boehringer Ingelheim and GlaxoSmithKline, all during the conduct of the study. L. Dupont has nothing to disclose. S.S. Birring reports grants from Merck, and personal fees (for scientific advisory work) from Merck, Bayer, Respivant, Pfizer and Sanofi, all outside the submitted work. J. Boyd is an employee of the European Lung Foundation. K.F. Chung has received honoraria for participating in advisory board meetings from GlaxoSmithKline, AstraZeneca, Novartis, Boehringer Ingelheim and Teva, regarding treatments for asthma and chronic obstructive pulmonary disease; and has also been renumerated for speaking engagements by AstraZeneca, Novartis and Merck. M. Dabrowska reports personal fees and other support from Angelini Pharma Poland (travel expenses and fees for attendance at ERS International Congresses 2017 and 2018 and at International Symposium on Cough 2018, and fees for lectures) and Chiesi (fees for attendance at ERS Congress 2016 and for lectures), outside the submitted work. C. Domingo reports personal fees (including speaker's honoraria) from ALK, Almirall, Chiesi, Esteve, Ferrer, GSK, Hal Allergy, ImmunoTek, Menarini, Sanofi, Novartis, Stallergenes Greer and Teva; non-financial support from GSK; and other support (including congress fees and advisory board fees) from Allergy Therapeutics, ALK, AstraZeneca, Menarini, Novartis, MSD and Teva, all outside the submitted work. G. Fontana has nothing to disclose. L. Guilleminault reports personal fees and non-financial support from ALK and AstraZeneca (for councils, invitations to congresses and participation in clinical trials) and from Chiesi (for a council and invitation to a congress); non-financial support from Boehringer Ingelheim (for invitation to a congress); personal fees from Bayer and GSK (for councils); other support from MSD (for participation in clinical trials); and personal fees, non-financial support and other support from Novartis (for councils, invitations to congresses and participation in clinical trials), all outside the submitted work. P. Kardos reports personal fees from AstraZeneca, GSK, Menarini, Novartis, Klosterfrau, Bionorica, Willmar Schwabe and MSD, and other support (for a phase 3 investigator cough study) from MSD, all outside the submitted work. E. Millqvist reports a pending user patent application for treatment of chronic cough with oral capsaicin. A.H. Morice reports grants from Merck, Bayer, GSK and Sanofi; and personal fees 
from Merck, Bayer, Bellus and Sanofi, all outside the submitted work. J.A. Smith reports grants from GlaxoSmithKline, Axalbion and Merck Inc. (funding for a commercial study to Manchester University NHS Foundation Trust), NeRRe Pharmaceuticals (to Christie Hospital, commercial funding for clinical trial), Menlo and Bayer (commercial funding for clinical trial), Afferent (to Manchester University NHS Foundation Trust) and AstraZeneca (contributed funding to MRC MICA grant); personal fees from GlaxoSmithKline and Merck Inc. (for advisory boards and consultancy work), from NeRRe Pharmaceuticals, Menlo, Bayer, Axalbion, AstraZeneca, Boehringer Ingelheim, Genentech, Neomed, Chiesi and Bellus (for consultancy), and from Afferent; and non-financial support from Vitalograph (for provision of cough monitoring equipment), all outside the submitted work. In addition, J.A. Smith is the inventor of a licensed patent "A method for generating output data" (owned by University Hospital of South Manchester, no personal royalties received). J.W. van den Berg reports personal fees and non-financial support (for a one-time advisory panel meeting) from MSD, outside the submitted work. C. Van de Kerkhove has nothing to disclose.

Support statement: J.A. Smith is funded by the National Institute for Health Research (NIHR) Manchester Biomedical Research Centre and is an NIHR Senior Investigator.

\section{References}

1 Chung KF, Pavord ID. Prevalence, pathogenesis, and causes of chronic cough. Lancet 2008; 371: 1364-1374.

2 Abdulqawi R, Dockry R, Holt K, et al. P2X3 receptor antagonist (AF-219) in refractory chronic cough: a randomised, double-blind, placebo-controlled phase 2 study. Lancet 2015; 385: 1198-1205.

3 Belvisi MG, Birrell MA, Wortley MA, et al. XEN-D0501, a novel transient receptor potential vanilloid 1 antagonist, does not reduce cough in patients with refractory cough. Am J Respir Crit Care Med 2017; 196: $1255-1263$.

4 Morice AH. TRPA1 receptors in chronic cough. Pulm Pharmacol Ther 2017; 47: 42-44.

5 Morice AH, McGarvey L, Pavord ID, et al. Theobromine for the treatment of persistent cough: a randomised, multicentre, double-blind, placebo-controlled clinical trial. J Thorac Dis 2017; 9: 1864-1872.

6 Smith JA, McGarvey LPA, Badri H, et al. Effects of a novel sodium channel blocker, GSK2339345, in patients with refractory chronic cough. Int J Clin Pharmacol Ther 2017; 55: 712-719.

7 Smith JA, Kitt MM, Morice AH, et al. MK-7264, a P2X3 receptor antagonist, reduces cough frequency in patients with refractory chronic cough: results from a randomized, controlled, phase $2 \mathrm{~b}$ clinical trial. Am J Respir Crit Care Med 2017; 195: A7608.

8 Morice AH, Jakes AD, Faruqi S, et al. A worldwide survey of chronic cough: a manifestation of enhanced somatosensory response. Eur Respir J 2014; 44: 1149-1155.

9 Chamberlain SA, Garrod R, Douiri A, et al. The impact of chronic cough: a cross-sectional European survey. Lung 2015; 193: 401-408.

10 French CT, Diekemper RL, Irwin RS, et al. Assessment of intervention fidelity and recommendations for researchers conducting studies on the diagnosis and treatment of chronic cough in the adult: CHEST guideline and expert panel report. Chest 2015; 148: 32-54.

11 Agusti A, Bel E, Thomas M, et al. Treatable traits: toward precision medicine of chronic airway diseases. Eur Respir J 2016; 47: 410-419.

12 Rennard S, Decramer M, Calverley PM, et al. Impact of COPD in North America and Europe in 2000: subjects' perspective of Confronting COPD International Survey. Eur Respir J 2002; 20: 799-805.

13 Kessler R, Partridge MR, Miravitlles M, et al. Symptom variability in patients with severe COPD: a pan-European cross-sectional study. Eur Respir J 2011; 37: 264-272.

14 Smith J, Woodcock A. Cough and its importance in COPD. Int I Chron Obstruct Pulmon Dis 2006; 1: 305-314.

15 Polley L, Yaman N, Heaney L, et al. Impact of cough across different chronic respiratory diseases: comparison of two cough-specific health-related quality of life questionnaires. Chest 2008; 134: 295-302.

16 Sinha A, Lee KK, Rafferty GF, et al. Predictors of objective cough frequency in pulmonary sarcoidosis. Eur Respir J 2016; 47: 1461-1471.

17 Ryerson CJ, Abbritti M, Ley B, et al. Cough predicts prognosis in idiopathic pulmonary fibrosis. Respirology 2011; 16: 969-975.

18 Smith SM, Schroeder K, Fahey T. Over-the-counter (OTC) medications for acute cough in children and adults in community settings. Cochrane Database Syst Rev 2014; 11: CD001831.

19 Chang $\mathrm{CC}$, Cheng $\mathrm{AC}$, Chang $\mathrm{AB}$. Over-the-counter (OTC) medications to reduce cough as an adjunct to antibiotics for acute pneumonia in children and adults. Cochrane Database Syst Rev 2014; 3: CD006088.

20 Dicpinigaitis PV. Cough: an unmet clinical need. Br J Pharmacol 2011; 163: 116-124.

21 Mazzone SB, Chung KF, McGarvey L. The heterogeneity of chronic cough: a case for endotypes of cough hypersensitivity. Lancet Respir Med 2018; 6: 636-646.

22 Chung KF, McGarvey L, Mazzone SB. Chronic cough as a neuropathic disorder. Lancet Respir Med 2013; 1: 414-422.

23 Morice AH, Millqvist E, Belvisi MG, et al. Expert opinion on the cough hypersensitivity syndrome in respiratory medicine. Eur Respir J 2014; 44: 1132-1148.

24 Undem BJ, Zaccone E, McGarvey L, et al. Neural dysfunction following respiratory viral infection as a cause of chronic cough hypersensitivity. Pulm Pharmacol Ther 2015; 33: 52-56.

25 Ando A, Farrell MJ, Mazzone SB. Cough-related neural processing in the brain: a roadmap for cough dysfunction? Neurosci Biobehav Rev 2014; 47: 457-468.

26 Bonvini SJ, Birrell MA, Smith JA, et al. Targeting TRP channels for chronic cough: from bench to bedside. Naunyn Schmiedebergs Arch Pharmacol 2015; 388: 401-420.

27 Chung KF. NMDA and GABA receptors as potential targets in cough hypersensitivity syndrome. Curr Opin Pharmacol 2015; 22: 29-36.

28 Pacheco A, Domingo C. Airway reflux: an emerging topic in respiratory medicine. Lancet Respir Med 2018; 6: $810-812$.

29 Ryan NM, Birring SS, Gibson PG. Gabapentin for refractory chronic cough: a randomised, double-blind, placebo-controlled trial. Lancet 2012; 380: 1583-1589. 
30 Kardos P, Dinh QT, Fuchs KH, et al. Leitlinie der Deutschen Gesellschaft für Pneumologie und Beatmungsmedizin zur Diagnostik und Therapie von erwachsenen Patienten mit Husten [Guidelines of the German Respiratory Society for diagnosis and treatment of adults suffering from acute, subacute and chronic cough]. Pneumologie 2019; 73: 143-180.

31 Pacheco A, de Diego A, Domingo C, et al. Chronic cough. Arch Bronconeumol 2015; 51: 579-589.

32 Morice AH, McGarvey L, Pavord I. Recommendations for the management of cough in adults. Thorax 2006; 61: Suppl. 1, i1-i24.

33 Société Française d'ORL et de Chirurgie de la face et du cou. Recommandations pour la pratique clinique: la toux chronique de l'adulte [Recommendations for clinical practice: chronic cough in the adult]. Rev Pneumol Clin 2006; 62: $271-281$.

34 Aliberti S, Polverino E, Chalmers JD, et al. The European Multicentre Bronchiectasis Audit and Research Collaboration (EMBARC) ERS Clinical Research Collaboration. Eur Respir J 2018; 52; 1802074.

35 Djukanovic R, Adcock IM, Anderson G, et al. The Severe Heterogeneous Asthma Research collaboration, Patient-centred (SHARP) ERS Clinical Research Collaboration: a new dawn in asthma research. Eur Respir J 2018; 52; 1801671.

36 Agusti A, Faner R, Donaldson G, et al. Chronic Airway Diseases Early Stratification (CADSET): a new ERS Clinical Research Collaboration. Eur Respir J 2019; 53; 1900217.

37 Cottin V, Annesi-Maesano I, Günther A, et al. The Ariane-IPF ERS Clinical Research Collaboration: seeking collaboration through launch of a federation of European registries on idiopathic pulmonary fibrosis. Eur Respir $\mathrm{J}$ 2019; 53: 1900539 